\title{
Number of \\ Detectable Kaon Decays at LAMPF II
}

T. W. L. Sanford

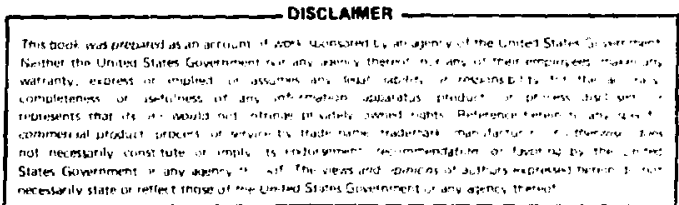




\title{
NUMBER OF DETECTABLE KAON DECAYS AT LAMPF II
}

by

$$
\text { T.W.L. Sanford }
$$

\begin{abstract}
The maximum number of kaon decays detectable at LAMPF II is estimated for both in-flight and stopping decays. Under reasonable assumptions, the momentum of the kaon beam that optimizes the decay yield occurs at about $6 \mathrm{GeV} / \mathrm{c}$ and $600 \mathrm{MeV} / \mathrm{c}$ for in-flight and stopping decays, respectively. $\mathrm{K}^{+}$decay yields are of the order of $7 \times 10^{\prime}$ per $10^{14}$ interacting protons with $K^{-}$yields being typically 5 times less.

By measuring decays from such beams, a statistical limit of $10^{-15}$ on a branching ratio to a particular channel can be placed in a 100-day run. The large number of kaon decays available at LAMPF II thus provides a powerful tool for sensitively examining rare-decay processes of the kaon.
\end{abstract}

\section{INTRODUCTION}

Experiments designed to measure kaon decays have been performed by measuring either secondaries from kaons decaying in flight (in-flight beam) or from kaons stopping in a target (stopping beam). A question that arises is, "What would an experimental configuration for measuring such decays look like at LAMPF II?" In the evaluation of this question, two fundamental quantities enter: (1) the maximum number of detectable kaon decays that are available from either an in-flight or stopping beam and (2) the momentum of the beam at which these ylelds are maximized.

In the following, these quantities are determined analytically by optinizing the kaon-decay yields with respect to kaon-decay monentun for two bean lines that have characteristics similar to ones currently operational at the BNL-AGS. 1 For the in-f1ight beam, the paraneters of the MESB bean are assuned : 


$$
\begin{aligned}
\frac{\Delta P}{P} & = \pm 3 \% \\
\Delta \Omega & =0.3 \mathrm{msr} \\
L & =45 \mathrm{~m}
\end{aligned}
$$

except that the nominal length of $81 \mathrm{~m}$ is reduced to $45 \mathrm{~m}$. For the stopping beam, the parameters of the LESB II are assumed:

$$
\begin{aligned}
& \frac{\Delta \mathrm{P}}{\mathrm{P}}= \pm 2.5 \% \\
& \Delta \Omega=15 \mathrm{msr} \\
& \mathrm{L}=15 \mathrm{~m} .
\end{aligned}
$$

The monentum of the incident proton beam is taken to be $16 \mathrm{GeV} / \mathrm{c}$ and the káon production angle is fixed at zero degrees. ${ }^{2}$

The optinization is based on the SW (Sanford-Wang) cross section ${ }^{3}$ with KR (kinematic reflection). ${ }^{4}$ Before the results of the In-flight and atopping optinization are presented, the SW cross section is discussed briefly.

II. SANFORD-WANG CROSS SECTIION

The SW cross section as a function of kaon momentum $P$ is written as:

$$
\left(\frac{d^{2} N}{d \Omega d P}\right) \quad s W=C_{1} P_{2}\left(1-\frac{P}{P_{1}}\right) \exp \left[-\frac{C_{3} P^{C_{4}}}{P^{C_{5}}}-C_{6} \theta\left(P-C_{7} P_{i} \cos C_{8 \theta}\right)\right] \text {, }
$$


where $P_{i}$ is the incident proton momentum and the parameters $c_{1}, c_{2}, \ldots, c_{8}$ are defined by

\begin{tabular}{|c|cccccccc|}
\hline & $\mathrm{C}_{1}$ & $\mathrm{c}_{2}$ & $\mathrm{C}_{3}$ & $\mathrm{C}_{4}$ & $\mathrm{c}_{5}$ & $\mathrm{c}_{6}$ & $\mathrm{c}_{7}$ & $\mathrm{c}_{8}$ \\
\hline $\mathbf{K}^{+}$ & 0.05897 & 0.6916 & 3.744 & 4.520 & 4.190 & 4.928 & 0.1922 & 50.28 \\
$\mathbf{K}^{-}$ & 0.02210 & 1.3230 & 9.671 & 1.712 & 1.643 & 4.673 & 0.1686 & 77.27 \\
\hline
\end{tabular}

For low momentum the cross section approaches

$$
\left(\frac{\mathrm{d}^{2} \mathrm{~N}}{\mathrm{~d} \Omega \mathrm{dP}}\right)_{S W} \rightarrow\left\{\begin{array}{l}
0.059 \mathrm{P}^{0.69} \mathrm{~K}^{+} \\
0.022 \mathrm{P}^{1.32} \mathrm{~K}^{-}
\end{array}\right.
$$

In this region, little experimental data existed at the time the $\mathrm{SW}$ parameterization was made. As such, the principle of $\mathrm{KR}$ is used to extend the range of validity of the $\mathrm{SW}$ cross section. Below about $1 \mathrm{GeV} / \mathrm{c}$ the cross section can be written

$$
\frac{d^{2} N}{d \Omega d P}=\left(\frac{d^{2} N}{d \Omega d P_{+}}\right) \text {SW } \frac{P^{2}}{E} \frac{E_{+}}{P_{+}^{2}},
$$

where $P_{+}$is defined from the figure:
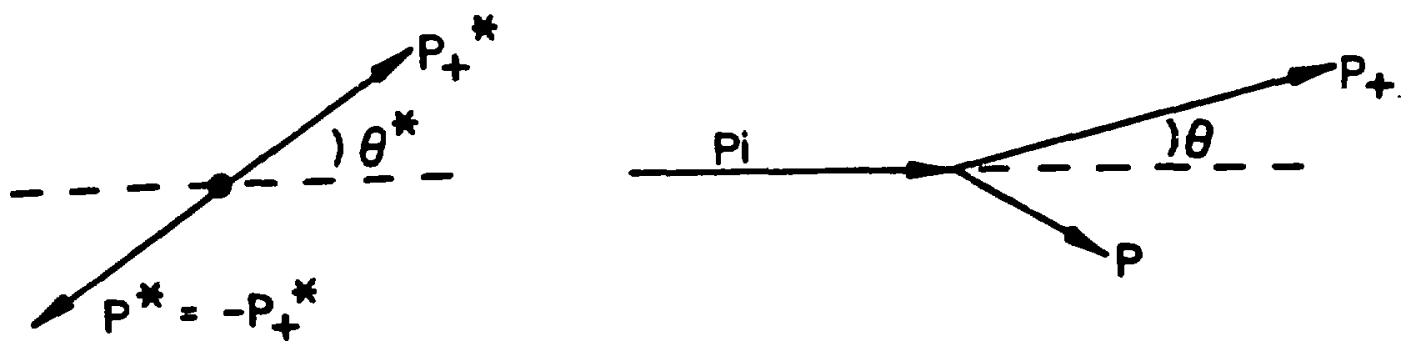
The expression gfives a stronger $P$ dependence than does the pure $S W$ cross section at low P:

$$
\frac{d^{2} N}{d \Omega d P}+c\left(\frac{d^{2} N}{d \Omega d P}\right) S W P^{2}
$$

Figure 1 shows results of the expression for 16-GeV/c incident proton energy. The peak of the $\mathrm{K}^{+} \mathrm{flux}$ occurs at $5 \mathrm{GeV} / \mathrm{c}$ and is three times that of the $\mathrm{K}^{-}$flux, which peaks at $3 \mathrm{GeV} / \mathrm{c}$.

A forward-traveling kaon with a $P_{+}$of $1.4 \mathrm{GeV} / \mathrm{c}$ in the laboratory has zero momentum in the $\mathrm{cm}$ frame. For $P_{+}$equal to $8.5 \mathrm{GeV} / \mathrm{c}$, the corresponding $P$ has zero momentum in the laboratory. The SW cross section between $1.4 \mathrm{GeV} / \mathrm{c}$ and 8.5 $\mathrm{GeV} / \mathrm{c}$ is thus used via the principle of KR to evaluate the cross section between $1.4 \mathrm{GeV} / \mathrm{c}$ and $0 \mathrm{GeV} / \mathrm{c}$, respectively.

As a side point, the maximum kaon momentum allowed for a $16-\mathrm{GeV} / \mathrm{c}$ incident proton $1 \mathrm{~s} 11.9 \mathrm{GeV} / \mathrm{c}$ if Fermi momentum be ignored.

The kaon data of $\mathrm{KEK}$ at $12.9 \mathrm{GeV} / \mathrm{c}$ incident-proton momentum ${ }^{5}$ provide a check on the absolute value of the SW cross section, for $K^{+}$and $K^{-}$mesons, as well as a check on the shape of the cross section below $2 \mathrm{GeV} / \mathrm{C}$. In Figs. 2 and 3 , the KEK data have been normalized to the SW cross section at 1.79 $\mathrm{GeV} / \mathrm{c}$. For the $\mathrm{K}^{+}$data, the normalization required increasing the measured cross section by $13 \%$, and for the $\mathrm{K}^{-}$data, the normalization required increasing the cross section by $30 \%$. These shifts are within the normalization uncertainty of the data. The two measurements at $735 \mathrm{MeV} / \mathrm{c}$ support the KR hypothesis.

\section{IN-FLIGHT BEAM}

A magnetic spectrometer is the typical apparatus for measuring in-flight decays. The aperture of the dipole employed in such a spectrometer is usually limited to $1 \mathrm{~m}$ in the direction of the field. The maximum monentim of secondaries from kaon decay in the $\mathrm{cm}$ frame or the equivalent maximum transverse-momentum $P_{T}$ in the laboratory is about $225 \mathrm{HeV} / \mathrm{c}$. Define $D$ by the following construction: 


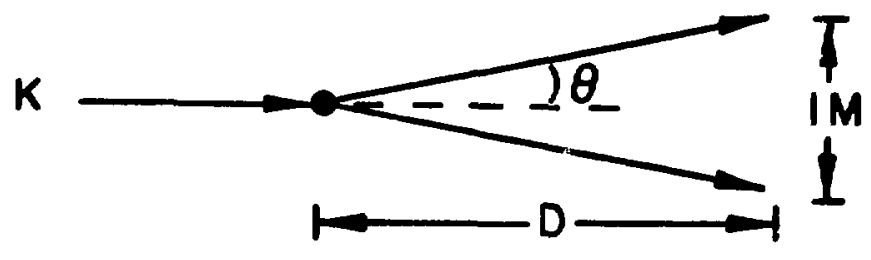

$$
\begin{aligned}
\theta & =\frac{0.5}{D[\mathrm{~m}]}=\frac{\mathrm{P}_{\mathrm{T}}}{\mathrm{P} / 2}=\frac{0.225}{\mathrm{P}[\mathrm{GeV} / \mathrm{c}] / 2} \\
D[\mathrm{M}] & =1.1 \times \mathrm{P}[\mathrm{GeV} / \mathrm{c}] .
\end{aligned}
$$

Then the secondaries from decays that occur within $D$ of the dipole can all be detected by the spectrometer. The width of the dipole is assumed to be at least $1 \mathrm{~m}$, and decays within any detector upstream of the dipoie are ignored.

Although it is expensive to inciease the aperture beyond $1 \mathrm{~m}$, no difficulty is encountered in allowing the decay volume to lengthen. Even at $9 \mathrm{GeV} / \mathrm{c}, \mathrm{D}$ is only $10 \mathrm{~m}$. If the length $D$ is taken as that which is characteristic for an experiment measuring decays in flight, then the acceptance is independent of kaon momentum and is roughly $14 \%$ :

$$
\begin{aligned}
& \left(\begin{array}{ll}
\left.C_{\tau}=3.71 \mathrm{~m} \quad \mathrm{~m}_{k}=0.494 \mathrm{GeV} / \mathrm{c}\right)
\end{array}\right. \\
& f=1-\exp \left(-\frac{D}{\frac{P_{C}}{m_{k}}}\right) \\
& \mathrm{f}=1-\exp \left(\frac{1.1 \times \mathrm{P}}{\frac{\mathrm{P}}{0.494} \times 3.71}\right)
\end{aligned}
$$

$=14 \%$. 
Often, the reconstructed kaon mass or missing transverse momenta are signiflcant constraints and are necessary in rejecting background. MCS (Multipole-Coulomb-Scattering) at low momentum and fixed angular resolution at high momentum cause the experimental mass resolution as measured in a spectrometer to broaden. The percentage background under the mass peak then increases and hence the sensitivity to a given decay decreases.

Neglecting these types of experimental considerations, the number of useful detectable decays is then:

$$
N(P)=f \times \frac{d N(P)}{d \Omega d P} \times e^{-L /\left(\frac{P}{m_{k}} \times C \tau\right)} \Delta P \Delta \Omega,
$$

where $\mathrm{L}$ is the length of the beam 1ine, $\Delta \mathrm{P}$ its momentum acceptance, and $\Delta \Omega$ its angular acceptance. Assuming that $\mathrm{L}$ is $45 \mathrm{~m}$ and that $\Delta \mathrm{P}$ and $\Delta \Omega$ are constant, the maximum yields occur at $6.8 \mathrm{GeV} / \mathrm{c}$ and $4.5 \mathrm{GeV} / \mathrm{c}$ for $\mathrm{k}^{+}$and $\mathrm{K}^{-}$decays, respectively (Fig. 4). At these momenta the $\mathrm{K}^{+}$yield is 5.4 times that of the $\mathrm{K}^{-}$yield.

In reality, $\Delta P / P$ remalns constant for a given beam line because bending angles and apertures are fixed. Under these conditions, the optimization occurs at a momentum about $1 \mathrm{GeV} / \mathrm{c}$ higher than at the above (Fig. 4). At this momentum, the yield is $10 \%$ higher than at the lower momentum for fixed $\Delta P / P$.

\section{STOPPING BEAM}

A stopping beam consists of a beam line, beam degrader, and target. The momentum spread of a beam line is about $3 \%$ of the momentum, and the straggling In the degrader and target is about $2 \%$ of the range. For a beam in the momentum range 0.5 to $1.5 \mathrm{GeV}$, the pions, kaons, and protons are well separat in range so that a thin target will suffice to stop the bulk of the kaons without directly selecting pions or protons.

The stopping flux in such a totally absorbing target is

$$
N\left(P ;=\frac{d^{2} N}{d \Omega d P} \times e^{-L /\left(\frac{P}{m_{k}} \times C \tau\right)} \times e^{-R(P) / \Lambda} \Delta P \Delta \Omega\right.
$$


Here, $L$ is again the length of the beam line, $\Lambda$ is the absorption length of the degrader material, and $R(P)$ is the length of the degrader necessary to stop a kaon of momentum P. MCS and subsequent leakage from the degrader are ignored. In addition, the absorption length is assumed to be independent of momentum. For carbon and lead, it is taken to be

$$
\Lambda_{\mathrm{K}}+= \begin{cases}253 \mathrm{gm} / \mathrm{cm}^{2} & \mathrm{C} \\ 655 \mathrm{gm} / \mathrm{cm}^{2} & P_{\mathrm{b}}\end{cases}
$$

and

$$
\Lambda_{K^{-}}=\left\{\begin{array}{l}
77.4 \mathrm{gm} / \mathrm{cm}^{2} \mathrm{C} \\
210 \mathrm{gm} / \mathrm{cm}^{2} P_{b}
\end{array}\right.
$$

for $\mathrm{K}^{+}$and $\mathrm{K}^{-}$, respectively. These lengths correspond to cross sections of

$$
\sigma_{\mathrm{K}}+\mathrm{A}^{2 / 3} \times 15 \mathrm{mb}
$$

and

$$
\sigma_{\mathrm{K}}^{-}=\mathrm{A}^{2 / 3} \times 48 \mathrm{mb}
$$

where $A$ is the atomic number. Below $2 \mathrm{GeV} / \mathrm{c}$ these cross sections are not constant, but the values used are a reasonable average over the variations encountered.

In practice, only stopping $\mathrm{K}^{+}$beams are used to measure kaon decays, because the $K^{-}$is absorbed before it has a chance to decay. Stopping $\mathrm{K}^{-}$beams are used, on the other hand, in the study of kaonic atoms.

The $\mathrm{K}^{-}$yields are shown in Figs. 5 and 6 for carbon and lead degraders. For carbon, the maximum yleld is reduced by a factor of 17 owing to decays along the 15-m beam line (Fig. 5). The effect of including KR in the SW cross section is to reduce the expected yield by $28 \%$ and to shift the optimun nomentun by only $50 \mathrm{MeV} / \mathrm{c}$, from $750 \mathrm{MeV} / \mathrm{c}$ to $800 \mathrm{MeV} / \mathrm{c}$. Figure 6 shows the effect of using different degraders. Yields vary because the kaon range is proportional to $1 / 2$, but the absorption length is proportional to $1 / \mathrm{A}^{2 / 3}$. If MCS $\mathrm{did}$ not reduce the flux and if background from nuclear interactions did not affect the given 
measurement, then the degrader with highest $\mathrm{A} / \mathrm{Z}$ ratio should produce the largest useful stopping flux. For lead, the peak of the yield occurs at $1040 \mathrm{MeV} / \mathrm{c}$, and the increase over that yield using a carbon degrader is 2.5 .

If the optimization is done keeping $\Delta \mathrm{P} / ?$ fixed, then the optimum momentum shifts upward by about $100 \mathrm{MeV} / \mathrm{c}$ for a net increase in yield of $10 \%$.

Maximum yields of $\mathrm{K}^{+}$occur at $1380 \mathrm{MeV} / \mathrm{c}$ and $1500 \mathrm{MeV} / \mathrm{c}$ for carbon and lead degraders, respectively, with the yield being twice as high if lead is used instead of carbon (Fig. 7). For $\mathrm{K}^{+}$, optimization occurs at a higher momentum than that for $\mathrm{K}^{-}$, because the absorption length is three times Ionger than for $\mathrm{K}^{-}$. If $48 \mathrm{mb} /$ nucleon were used instead of $15 \mathrm{mb} /$ nucleon, then the $\mathrm{K}^{+}$spectrum would have the same shape as the $K^{-}$spectrum. For carbon, the peak would shift from $1380 \mathrm{MeV} / \mathrm{c}$ to $800 \mathrm{MeV} / \mathrm{c}$ with a decrease of $10 \mathrm{in}$ yield.

At the optimum momenta for the lead degrader, $71 \%$ and $87 \%$ of the $\mathrm{K}^{+}$and $\mathrm{K}^{-}$ interact and are absorbed (Fig. 8). The backgrounds generated by these unwanted interactions may severely limit the performance of a given stopping experinent. Let us assume, for the purpose of this discussion, that the maximum useful yield is limited at the point at which the degrader absorbs $50 \%$ of the incident flux. On the basis of this assumption, the optimum momenta drop from $1500 \mathrm{MeV} / \mathrm{c}$ to $1035 \mathrm{MeV} / \mathrm{c}$ and from $800 \mathrm{MeV} / \mathrm{c}$ to $625 \mathrm{MeV} / \mathrm{c}$, with decreases in yields of 1.3 and 2.7 for the $\mathrm{K}^{+}$and $\mathrm{K}^{-}$bearn, respectively.

Even though we are dealing with separated kaon beams, there may be substantial pion contamination. Pions have an absorption length closer to that of a $\mathrm{K}^{-}$rather than to that of a $\mathrm{K}^{+}$. Accordingly, depending on the fraction of the contamination, the degrader for the $\mathrm{K}^{+}$beam may also be 1 imited to a thickness similar to that which limits the $\mathrm{K}^{-}$beam.

\section{SUMMARY}

The agreement of the SW cross section using KR with the KEK data at 12.9 $\mathrm{GeV} / \mathrm{C}$ gives confidence that the SW cross section with KR can be used to predict cross sections at $16 \mathrm{GeV} / \mathrm{c}$. In proceeding from these cross sections to estimating the maximum number of useful kaon decays, many simplifying assumptions have been made. The assumptions are reasonable, however, so that numbers in the following tables do give a rough guide as to the aaximum kaon-decay ylelds that are possible at LAMPF II. 
The main assumptions, aside from beam-1ine constraints, are that 147 of the in-flight decays are detectable, that the degrader length is limited by the requirement that no more than $50 \%$ of the beam be absorbed, and that $50 \%$ of the stopping decays are detectable. Under these conditions the maximum detectable kaon-decay yields occur at the following momenta:

\begin{tabular}{|c|c|c|}
\hline & In-Flight & Stopping (Lead) \\
\hline $\mathrm{K}^{+}$ & $\begin{array}{c}\mathrm{GeV} / \mathrm{c} \\
6.8\end{array}$ & $\begin{array}{c}\mathrm{GeV} / \mathrm{c} \\
1.0 \\
\ddagger \\
0.63\end{array}$ \\
\hline $\mathrm{K}^{-}$ & 4.5 & 0.63 \\
\hline
\end{tabular}

The optimum $\mathrm{K}^{+}$momentum for the stopping beam depends on the pion contamination. The $1 \mathrm{GeV} / \mathrm{c}$ optimum corresponds to a beam of pure positive kaons, whereas the $0.63 \mathrm{GeV} / \mathrm{c}$ optimum corresponds to a beam with a large pion contamination. At the above momenta, the kaon-decay yields per $\Delta P \Delta \Omega$ and per interacting proton are:

\begin{tabular}{|c|c|c|}
\hline & In-Flight & Stopping (Lead) \\
\hline$K^{+}$ & $\begin{array}{l}\text { Yield/SR/GeV/Int. P } \\
6.0 \times 10^{-3}\end{array}$ & $\begin{array}{c}\text { Yield/SR/GeV/Int. P } \\
1.6 \times 10^{-3} \\
\begin{array}{l}\ddagger \\
2.8 \times 10^{-4}\end{array}\end{array}$ \\
\hline $\mathrm{K}^{-}$ & $1.1 \times 10^{-3}$ & $6.8 \times 10^{-5}$ \\
\hline
\end{tabular}

Using the parameters of the LESB II and MESB beams, the maximum detectable yields per $10^{14}$ interacting protons are:

\begin{tabular}{|c|c|c|}
\hline & MESB & LESB II \\
\hline $\mathrm{K}^{+}$ & $\begin{array}{c}\text { Yield } / 10^{14} \text { Int. P } \\
7.3 \times 10^{7}\end{array}$ & $\begin{array}{r}\text { Yie1d } / 10^{14} \text { Int. } P \\
1.8 \times 10^{8} \\
\ddagger \\
3.2 \times 10^{7}\end{array}$ \\
\hline$k^{-}$ & $1.3 \times 10^{7}$ & $7.5 \times 10^{6}$ \\
\hline
\end{tabular}


The $k^{+} / K^{-}$yield ratios are

\begin{tabular}{|c|c|c|}
\hline & MESB & LESB II \\
\hline $\mathrm{K}^{+} / \mathrm{K}^{-}$ & 5.6 & 24 \\
& & $\uparrow$ \\
& & 4.1 \\
\hline
\end{tabular}

and the yields of the MESB beam with respect to the LESB II beam are

\begin{tabular}{|l|l|l|}
\hline & $\mathrm{K}^{+}$ & $\mathrm{K}^{-}$ \\
\hline MESB/LESB II & $0_{\substack{0.41 \\
i_{+}^{+}}}^{2.4}$ & 1.7 \\
\hline
\end{tabular}

These ratios increase if MCS and background generation in the degrader are also taken into account.

Finally, consider the number of decays from the medium-energy beam of positive kaons. Assuming $10^{14}$ interacting protons per second, one could in principle be sensitive to $7 \times 10^{14}$ decays in a 100-day run:

$$
\mathrm{N}=10^{2} \text { day } \cdot 10^{5} \frac{\mathrm{s}}{\mathrm{day}} \cdot 7 \times 10^{7} \frac{\mathrm{k}^{+}}{\mathrm{s}}=7 \times 10^{14}
$$

A statistical limit of $1.4 \times 10^{-15}$ on a branching ratio for a particular channel could then be placed. Comparable sensitivity is expected using the stopping beam. The large number of kaon decays avallable at LAMPF II from either an inflight or stopping beam thus provides a powerful tool for sensitively examining the rare-decay processes of the kaon.

\section{ACKNOWLEDGMENTS}

I would like to thank David Bowman, Cyrus Hoffman, and Robert Macek for many useful discussions concerning the contents of th.s note. 


\section{REFERENCES}

1. G. M. Bunce, "AGS Beams - May 1978," Brookhaven National Laboratory report BNL 50878 (1978).

2. For simplicity zero degrees is selected, and the variation of cross section with angle in the beam acceptance and absorption in the target are ignored. Because of absorption, maximum kaon-fluxes actually occur for small but nonzero production angles. See, for example, D. Berley, "Optimizing Kaon Production," pp. 257-277 in Proceedings of the Summer Study Meeting on Kaon Physics and Facilities, Brookhaven National Laboratory report BNL 50579 (December 1976).

3. J. R. Sanford and C. L. Wang, "Empirical Formula for Particle Production in P-Be Collisions between 20 and $35 \mathrm{BeV} / \mathrm{C}$ Part II," Brookhaven National Laboratory report BNL-11479 (May 1967).

4. C. M. Hoffman, "How to Build a Very Low Momentum $K^{-}$Beam at a Kaon Factory," Los Alamos Nationa1 Laboratory report LA-8949-MS (August 1981).

5. A. Yamamoto, "Study on Low Energy Intense Kaon Beam," KEK Nationa1 Laboratory for High Energy Physics report KEK 81-13 (November 1981). 


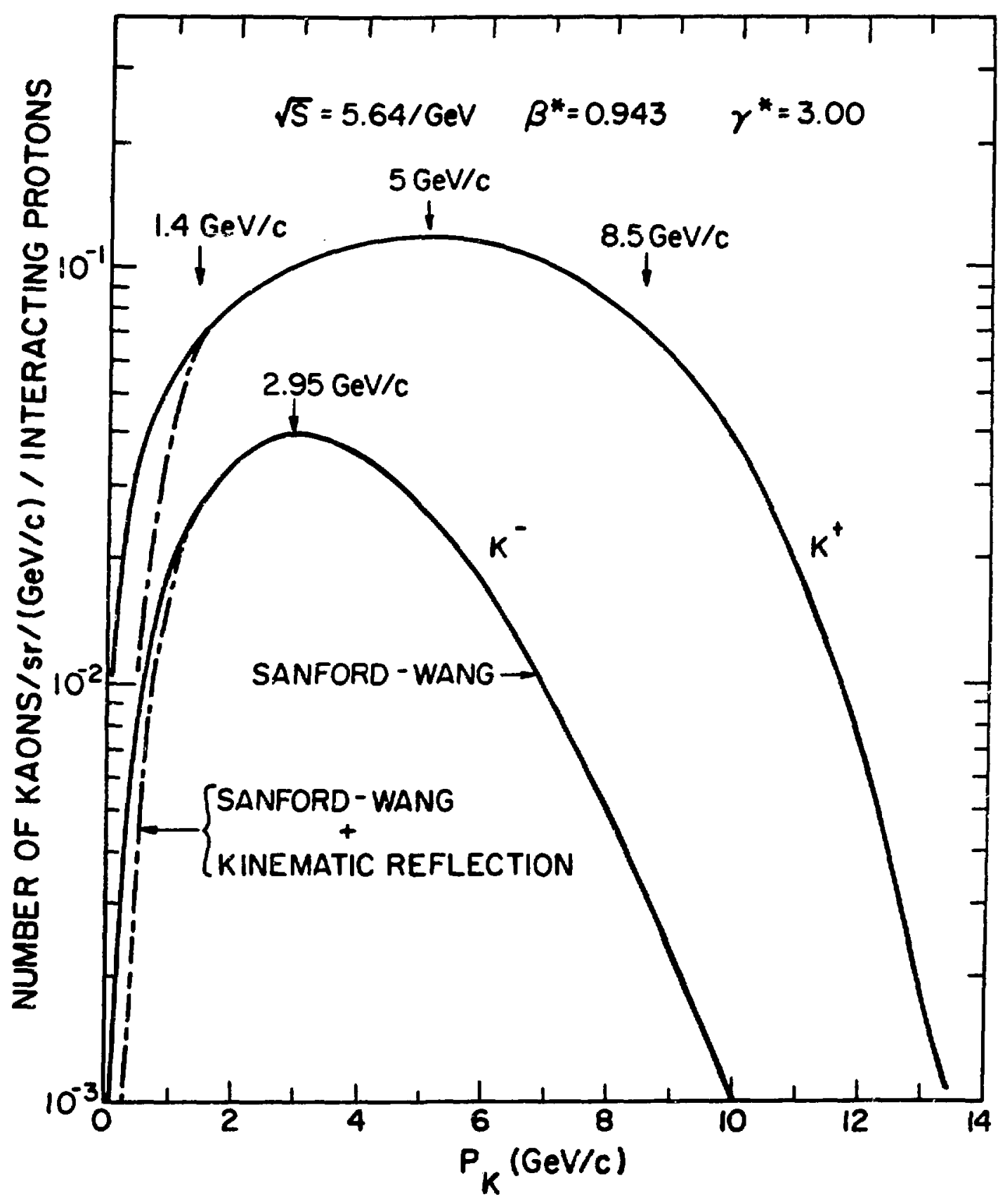

Fig. 1. Kaon production cross section at $0^{\circ}$ in $\mathrm{p}-\mathrm{Be}$ collisions at $16 \mathrm{GeV} / \mathrm{C}$. 


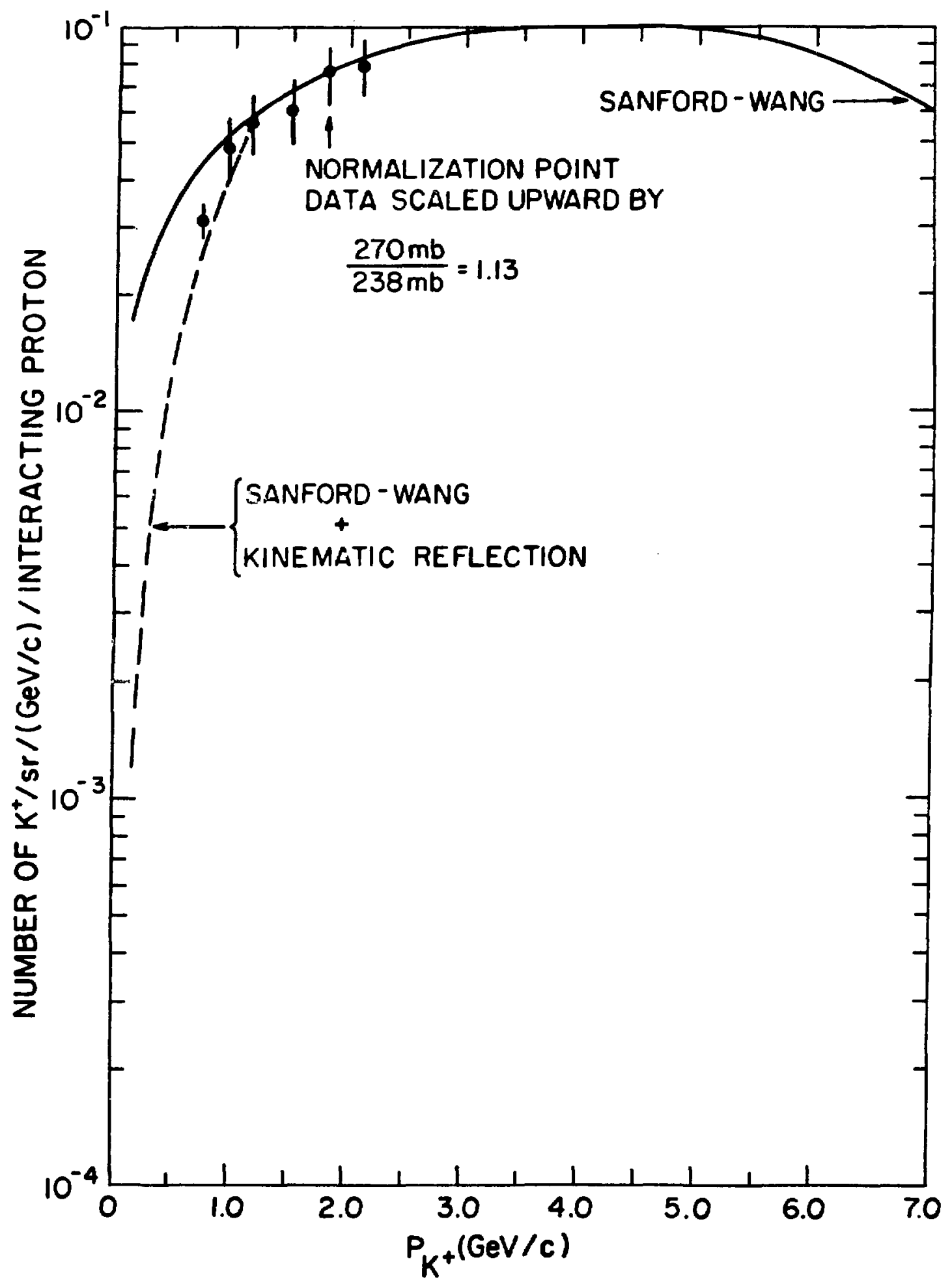

Fig. 2. Comparison of SW cross section with KEK data for $\mathrm{K}^{+}$production at $0^{\circ}$ in $\mathrm{P}$ Be collisions at $12.9 \mathrm{GeV} / \mathrm{c}$. 


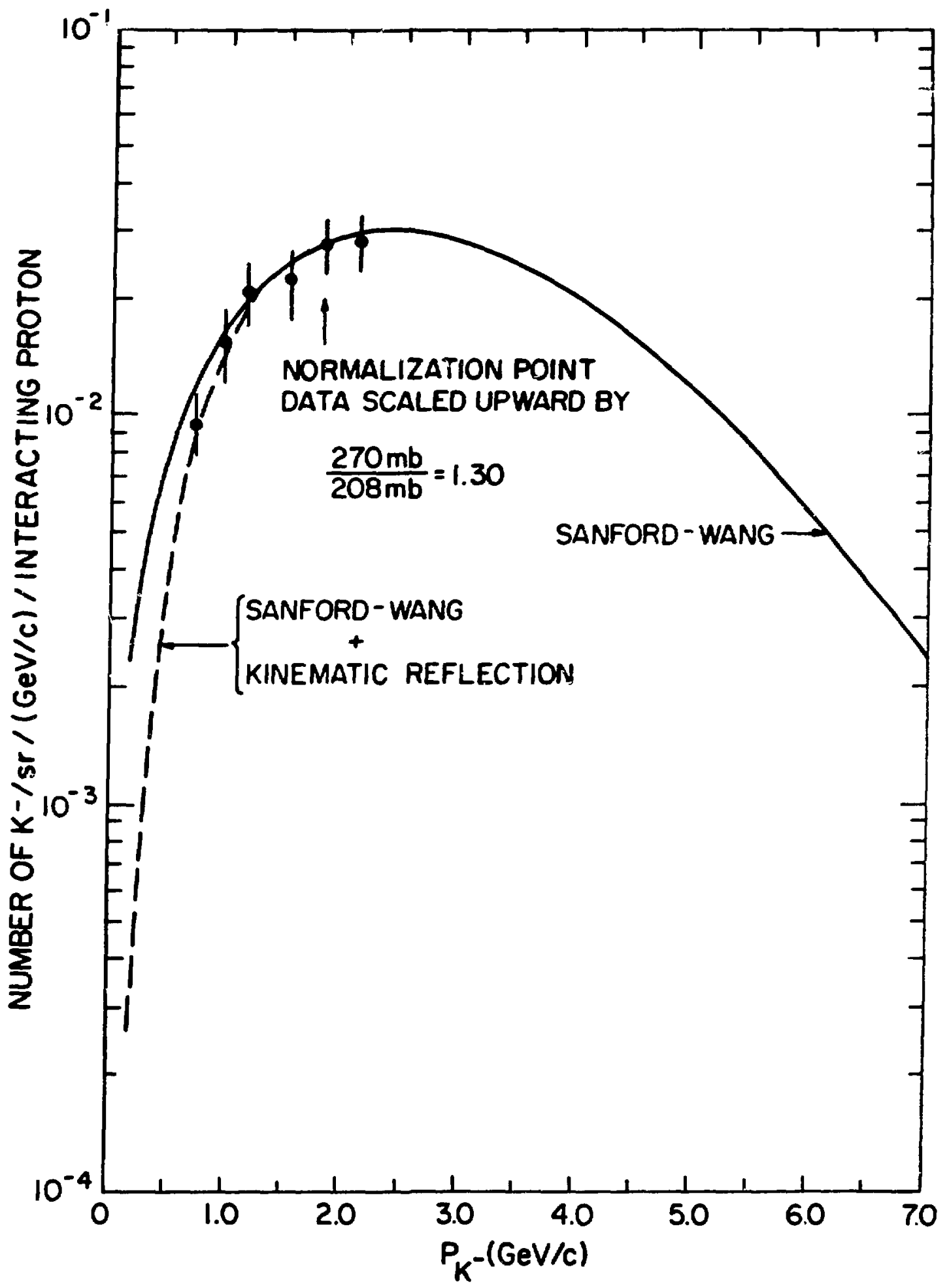

Fig. 3. Comparison of $\mathrm{SW}$ cross section with $\mathrm{KEK}$ data for $\mathrm{K}^{-}$production at $0^{\circ}$ in $\mathrm{p}-\mathrm{Be}$ collisions at $12.9 \mathrm{GeV} / \mathrm{c}$. 


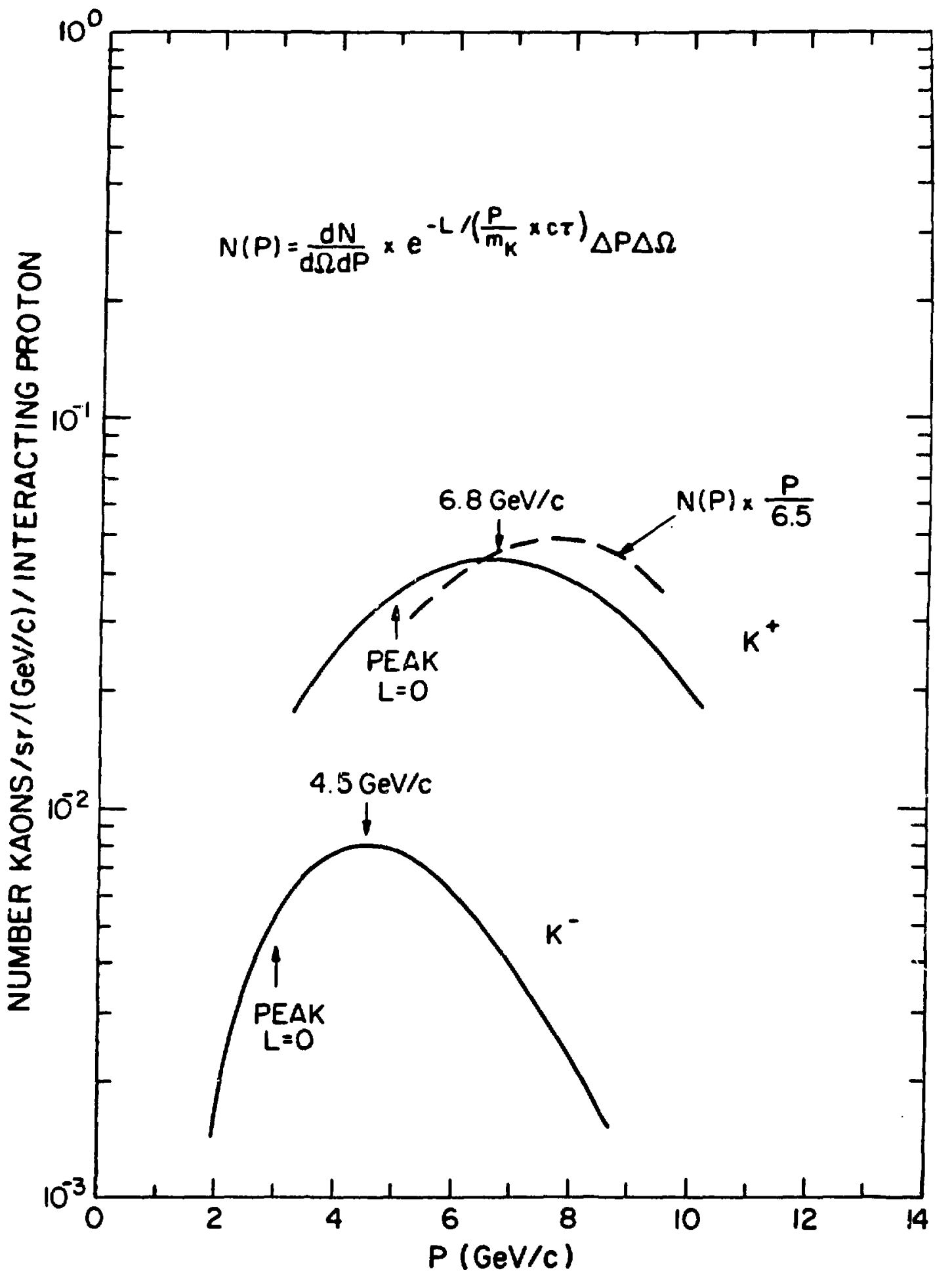

FIg. 4. Number of kaons at the extt of a 45-m beam 1ine. 


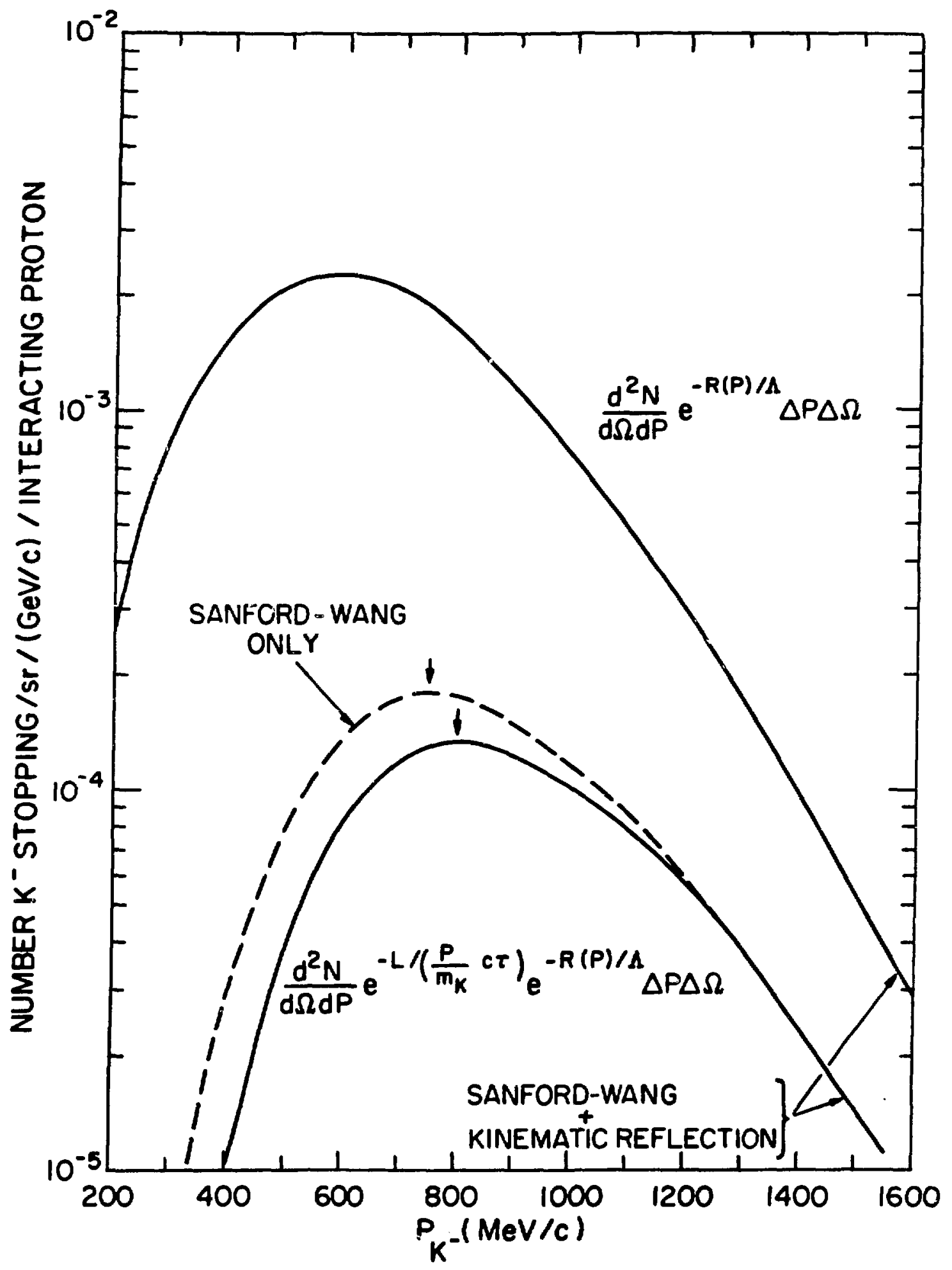

Fig. 5. Number of $\mathrm{K}^{-}$stopping using a carbon degrader for a 15-m beam 1ine. 


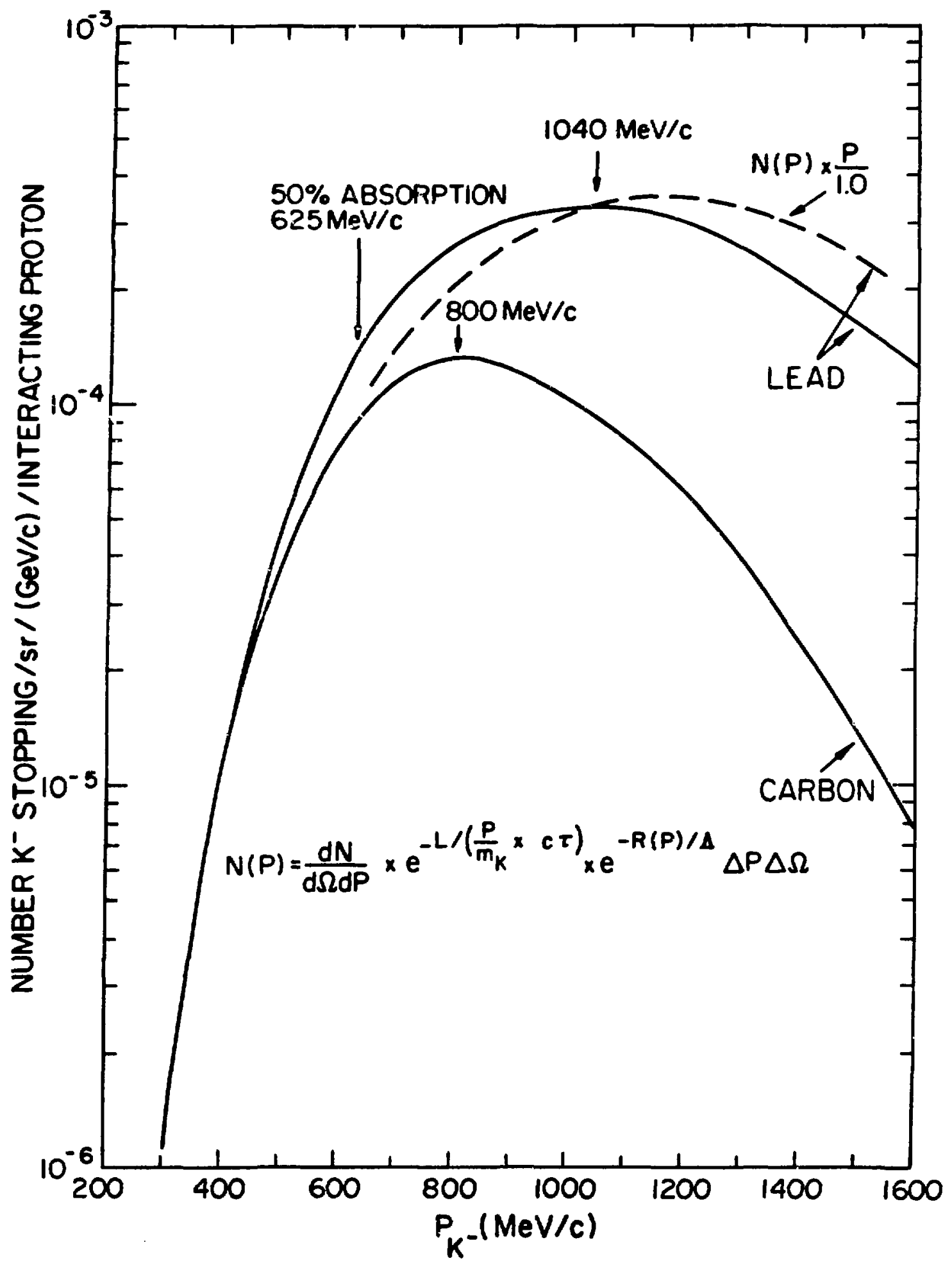

Fig. 6. Number of $\mathrm{K}^{-}$stopping using either carbon or lead degrader for a 15-m beam line. 


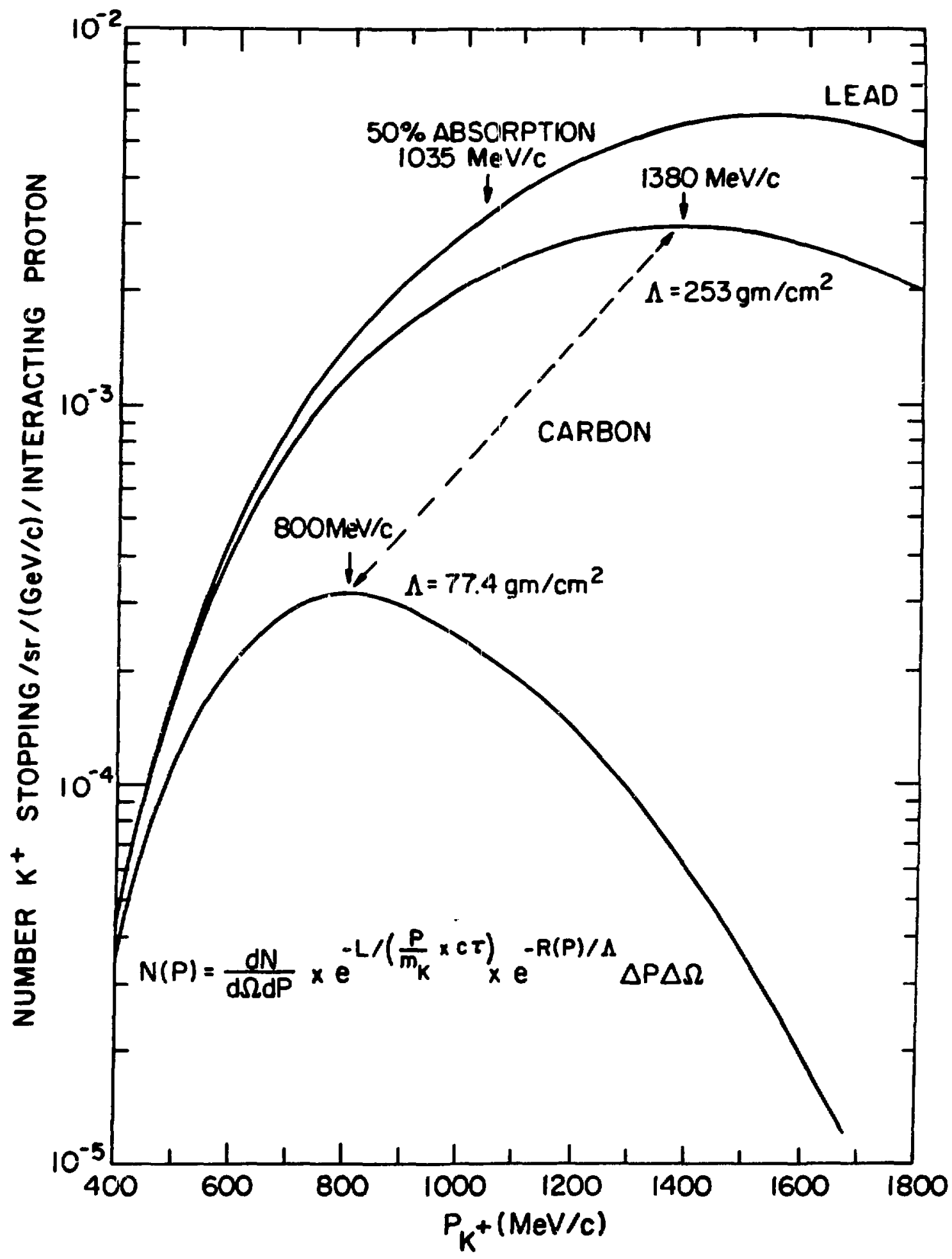

Fig. 7. Number of $\mathrm{K}^{+}$stopping using etther carbon or lead degrader for a $15-\mathrm{m}$ beam Iine. 


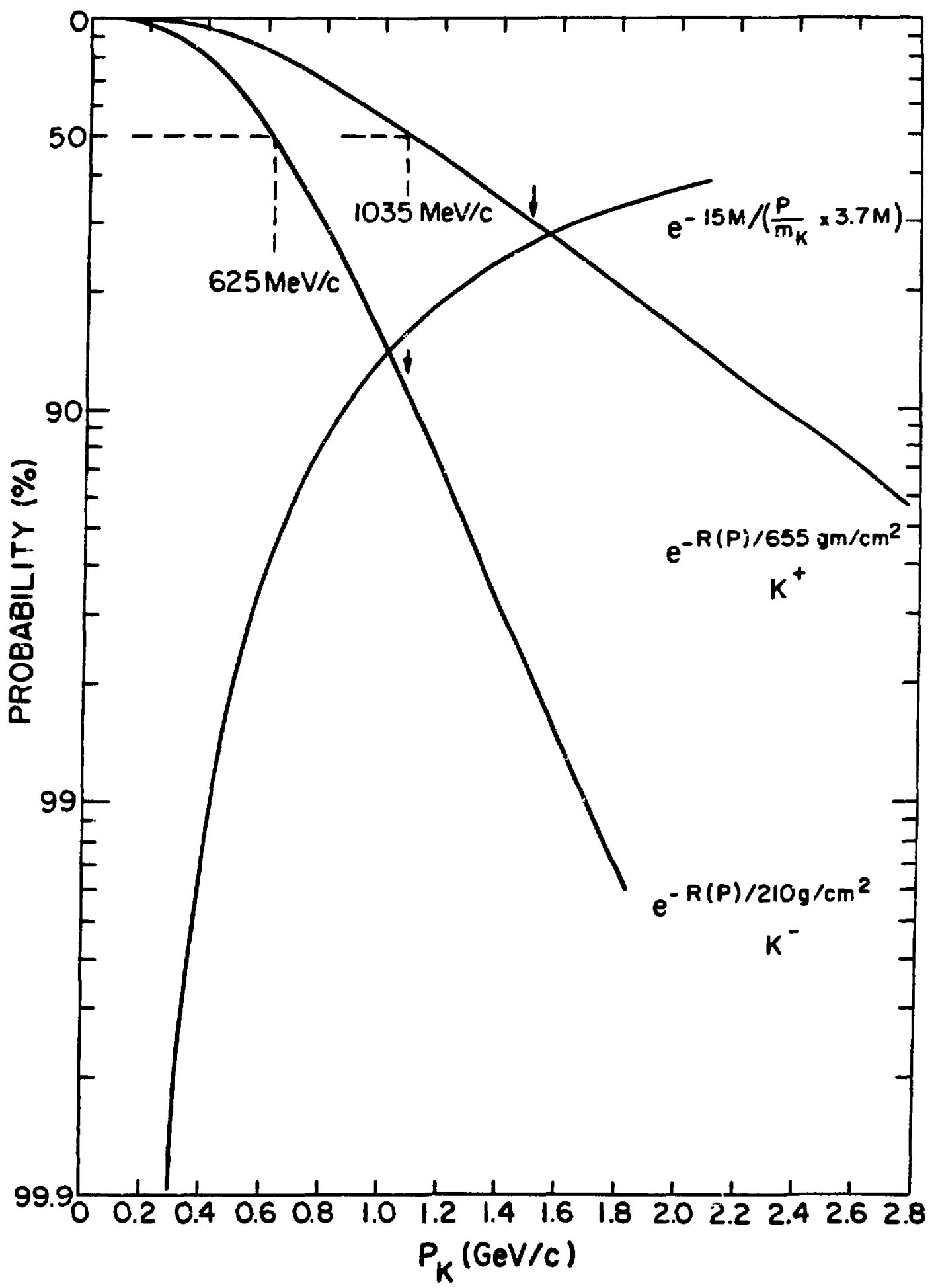

F1g. 8. Absorption in lead and decay probability versus $P_{K}, R(P)$ is the kaon range in lead. 